\title{
Cognitive biases predict symptoms of depression, anxiety and wellbeing above and beyond neuroticism in adolescence
}

Article

Accepted Version

Creative Commons: Attribution-Noncommercial-No Derivative Works 4.0

Smith, E. M., Reynolds, S., Orchard, F., Whalley, H. C. and Chan, S. W. Y. (2018) Cognitive biases predict symptoms of depression, anxiety and wellbeing above and beyond neuroticism in adolescence. Journal of Affective Disorders, 241. pp. 446-453. ISSN 0165-0327 doi:

https://doi.org/10.1016/j.jad.2018.08.051 Available at https://centaur.reading.ac.uk/78568/

It is advisable to refer to the publisher's version if you intend to cite from the work. See Guidance on citing.

To link to this article DOI: http://dx.doi.org/10.1016/j.jad.2018.08.051

Publisher: Elsevier

All outputs in CentAUR are protected by Intellectual Property Rights law, including copyright law. Copyright and IPR is retained by the creators or other copyright holders. Terms and conditions for use of this material are defined in the End User Agreement. 


\section{CentAUR}

Central Archive at the University of Reading

Reading's research outputs online 
Word count: 4,472 (exl figures and ref)

Abstract: 261

No of figures: 1

No of tables: 3

Cognitive biases predict symptoms of depression, anxiety and wellbeing above and beyond neuroticism in adolescence

Eilidh M Smith ${ }^{1}$ Shirley Reynolds ${ }^{2}$, Faith Orchard², Heather C Whalley ${ }^{\star 1}$, Stella WY Chan $* \# 1$

Affiliations: University of Edinburgh ${ }^{1}$, University of Reading ${ }^{2}$

*: joint last authors

\# Corresponding author

Stella Chan

Department of Clinical Psychology

University of Edinburgh

Doorway 6 Medical Quad

Teviot Place

Edinburgh EH8 9AG

UK

Telephone: + 44 (0)1316513935

Email: stella.chan@ed.ac.uk

Keywords: Adolescence; Cognition; Anxiety; Depression; Wellbeing; Neuroticism 


\begin{abstract}
Background: Adolescence represents a period of vulnerability to affective disorders. Neuroticism is considered a heritable risk factor for depression, but is not directly amenable to intervention. Therefore, it is important to identify the contributions of modifiable risk factors. Negative cognitive biases are implicated in the onset and maintenance of affective disorders in adults, and may represent modifiable risk factors in adolescence.
\end{abstract}

Aim(s): This study sought to assess to what extent cognitive biases are able to predict depression, anxiety and wellbeing beyond that of neuroticism in adolescents. Methods: Adolescents $(\mathrm{N}=99)$ were recruited from Scottish secondary schools (54.5\% female; mean age=14.7). Cognitive biases of autobiographical memory, selfreferential memory, ambiguous scenarios interpretation, facial expression recognition, rumination and dysfunctional attitudes were assessed. Depression, anxiety, and wellbeing were indexed using the Mood and Feelings Questionnaire, Spence Children's Anxiety Scale and the BBC Subjective Wellbeing Scale respectively.

Results: Regression analyses demonstrated neuroticism to significantly predict depression, anxiety and wellbeing. The addition of cognitive biases resulted in a significant increase of explained variance with final models explaining just over $50 \%$ of variances of depression, anxiety and wellbeing. Conclusion: This demonstrates that cognitive biases explain mental health symptoms over and above that of the stable risk factor neuroticism. Depressive symptomology was particularly related to self-referential memory bias, while anxiety was predicted by interpretive bias. Results may inform treatment, targeting specific 
biases based on diagnostic features may be of benefit in alleviating distress and promoting wellbeing. 


\section{Introduction}

Depression is the leading cause of disability worldwide (WHO, 2017). Research shows that mental health disorders, including depression and anxiety, originate in adolescence (MQ, 2016). Lifetime prevalence in adolescents (13-18-year olds) was reported to be $11 \%$ for depression and $32 \%$ for anxiety (Avenevoli et al., 2015; Merikangas et al., 2010). Importantly, earlier onset of depression predicts longer episodes, more severe course, poorer recovery and higher recurrence rates (Dunn and Goodyer; 2006). In addition, it is important to consider positive measures of wellbeing and quality of life in combination with psychopathology and distress. Mental health is an integral component of wellbeing; in particular, wellbeing is considered to be greater than the absence of mental illness (World Health Organisation (WHO), 2013). Traditional emphasis on disorder and distress neglects the importance of wellbeing factors and positive measures of quality of life.

Adolescence represents a sensitive neurodevelopmental window for the fostering of lifelong positive mental health (Marco, Macri and Laviola, 2011). Therefore, there is an urgent need to understand risk factors that are specific to the adolescent period. A major risk factor for anxiety and depression is neuroticism (Navrady et al., 2017). Neuroticism has been considered a means of indexing risk and a general risk factor influencing the onset and course of psychological disorders (Klein et al., 2011). Lahey (2009) provided a discussion regarding the powerful predictive value of neuroticism in relation to longevity, psychiatric and physical health disorders.

Evidence supports a biological basis of neuroticism which is considered to be a largely heritable trait, sharing genetic factors underlying risk for internalising disorders (Lucinao et al., 2018, Hettema, et al., 2006). However, there is mixed 
evidence regarding the stability of neuroticism over time. Wray et al., (2007) examined genetic and environmental contributions to neuroticism across 22 years in over 20,000 individuals, to demonstrate that genetic correlations between measures over time were very high. However, environmental contributions demonstrated lower stability over time (Wray et al., 2007). Additionally, neuroticism has been shown to reduce following antidepressant administration (Tang et al., 2009), suggesting that the impact of improving mood through treatment may also have a more general impact affecting neuroticism. However, other interventions, such as cognitive, treatments have not demonstrated changes in neuroticism (Tang et al., 2009).

As neuroticism is not universally considered modifiable, it is important to consider mechanisms above and beyond neuroticism that may be more amenable to intervention. Cognitive factors have been targeted by mainstream treatments such as cognitive behavioural therapy (CBT; NICE 2005). However, CBT has demonstrated limited success in adolescents (Goodyer et al., 2017). It may be that CBT is less efficacious due to ongoing neurocognitive development and environmental factors specific to adolescence. As such, the identification of cognitive features that predict depression in this age range may help improve interventions with the potential for lifelong impact.

Negative attributional style and rumination are cognitive processes that have frequently been related to disorders. Meta-analyses consistently demonstrate that rumination is predictive of the onset, severity and course of symptoms of depression (Mor and Winquist, 2002; Nolen-Hoeksema, 2000). Similarly, negative attributional style is considered to create a vulnerability to depression (Lee and Hankin, 2009). In adolescents, McLaughlin and Nolen-Hoeksema (2011) demonstrated rumination fully 
mediated associations between anxiety and depression, identifying it as a transdiagnostic risk factor and target for intervention.

Negative cognitive biases of information processing have been proposed as mechanisms underlying the vulnerability, onset and maintenance of depression. Williams et al., (1997) proposes that negatively biased elaborative processing characterises depression. This necessitates increased allocation of cognitive resources to negative materials, resulting in the encoding of negative elaborations to memory, thereby enhancing memory for depression related materials. Previous research has examined cognitive biases in depression in adults and identified the importance of assessing biases across multiple domains of processing, a key limitation of research amongst adolescents (Everaert et al., 2015).

\section{Autobiographical Memory}

Impairment of specific autobiographical memory recall has been robustly associated with adult depression (Williams et al., 1997). Overgeneral memory refers to a memory which lacks specificity and lasts an extended duration (Williams et al., 1997) Sumner et al., (2011) implicated overgeneral memory in the onset of depression, demonstrating that overgeneral memory retrieval was predictive of depressive relapse in adolescents experiencing chronic interpersonal stress. However, studies of autobiographical memory and depression in adolescents, including clinical, community and at-risk samples, have produced mixed findings (Swales et al., 2010; Chan et al., 2007). Overall, there is a lack of consensus regarding the context and mechanisms of autobiographical memory performance and the majority of previous studies were based on adult patient samples.

\section{Self-Referential Memory}


Biased self-referential memory has also been implicated in emotional disorders. A study of depressed youth employed a self-referent encoding task to demonstrate memory bias in the recall of negative compared to positive words (Zupan et al., 1987). However, Timbremont et al., (2008) found no differences in memory biases between currently, never and previously depressed youth. A recent review (Platt et al., 2017) concluded that studies using self-referent encoding tasks have produced mixed findings and evidence of negative biases is inconclusive across clinical, at risk and community samples.

\section{$\underline{\text { Interpretation Bias }}$}

Negatively biased interpretation has also been associated with depression in adolescents. Orchard et al., (2016) demonstrated that adolescents with depression make significantly more negative interpretations of ambiguous scenarios than nondepressed patient and community control groups. However, Micco et al., (2014) showed that interpretation bias modification reduced negative biases in depressed and control groups of adolescents, but there was no associated change in anxiety or depression. Again, overall conclusions are limited by the scarcity of studies in the adolescent population.

\section{Biased Processing of Emotional Facial Expressions}

Empirical evidence of deficits or biases in emotional processing of facial expressions associated with adolescent depression are particularly inconsistent. Schepman et al., (2011) found no deficits of accuracy amongst depressed adolescents, although the depressed group demonstrated a negative bias at low intensities compared to controls. Contrastingly, Joormann et al., (2010) demonstrated impaired accuracy of identification and that children at high familial risk for depression required greater 
emotional intensity of facial stimuli to accurately identify sad facial expressions. These inconsistent results could be due to varying methodology.

While there is substantial, although mixed, research supporting the relationship between cognitive biases and depression in adults, the adolescent population has been less studied and understanding of cognitive biases within this age group is limited. Furthermore, few studies have assessed multiple realms of biases within the same sample, this may account for discrepancy within results. This study aimed to address this limitation by assessing multiple realms of processing within the same sample.

The high prevalence of mental ill-health within the adolescent population (Avenevoli et al., 2015), alongside the conceptualisation of mental health as a spectrum indicates that symptoms are likely present within individuals recruited from community settings. Furthermore, Wang et al., (2005) found a median 8-year delay between symptom onset and contact with services. Therefore, sampling restricted to patient groups risks overlooking individuals who are not help-seeking, those who may be demonstrating pre-clinical or sub-diagnostic symptoms, or those exhibiting resilience factors.

To achieve a representative sample, this study therefore aimed to recruit adolescents from community settings to examine to what extent cognitive processes (attribution style, rumination and cognitive biases) are able to predict three outcome variables (depression, anxiety and wellbeing) within adolescents. Secondly, to consider the contribution of cognitive processes in relation to neuroticism. This study has examined contributions of cognitive bias factors independently from neuroticism in order to distinguish the influence of cognitive factors distinct from underlying 
personality risk. We hypothesised that cognitive measures will predict depression, anxiety and wellbeing above that of neuroticism in adolescence, and that there may be distinct contributions of biases to depression, anxiety and wellbeing.

\section{Methodology}

\section{Participants}

Inconsistencies exist within the research literature as to the age range distinguishing the 'adolescent' period. This developmental stage is associated with social and neurological changes as well as related to an increased risk for psychiatric disorderz (Caasey et al., 2008; Kessler et al., 2005). In line with previous research this study sought to recruit adolescents aged $12-18$ years (mean=14.9, S.D. $=1.52, \mathrm{~N}=99$ ) from Scottish secondary schools (58.6\% female and $70.3 \%$ White British; Table 1$)$. A power calculation determined that the current sample size allowed for up to 6 predictors in a regression model with $80 \%$ power to detect a medium effect size.

\section{Measures}

All of the following standardized measures have been used in previous research with adolescents and considered valid and reliable. Their reliability in this sample has been confirmed with high Cronbach's alphas, as reported below. Depressive symptoms were assessed using the Mood and Feelings Questionnaire (Child Version; MFQ; Angold and Costello; 1987; $\alpha=.91$ ). Anxiety symptoms were assessed using the Spence Children's Anxiety Scale (SCAS; Spence; 1998; $\alpha=.89$ ). Wellbeing was assessed using the BBC Well-being Scale (BBC; Kinderman, Schwannauer, Pontin and Tai; 2011; $\alpha=.95)$. Neuroticism was measured with the 12-item short 
version of Eysenck Personality Questionnaire-Neuroticism subscale (EPQ-N; Eysenck et al., 1985; $\alpha=.83$ ). The Ruminative Response Styles scale (RRS; Treynor et al., 2003; $\alpha=.87$ ) measured rumination; higher scores indicate more ruminative thinking. Dysfunctional attribution style was measured with the Dysfunctional Attitudes Scale: 24 Item Version (DAS-24; Power et al., 1994; $\alpha=.80$ ) with lower scores indicating more dysfunctional thoughts.

\section{Self-Reference Recall Task (Kelvin et al. 1999)}

This task assessed biases of self-referential memory recall and consisted of 12 positive and 12 negative adjectives. This task has been previously employed in research literature and significant differences in this task were demonstrated in participants before and after negative mood induction (Kelvin et al.,1999). Participants indicated the extent to which each word described them on a four-point scale (1='not at all like me', $4=$ ='very much like me'). To counter primacy and recency effects, three neutral adjectives were included at the beginning and end, which were excluded from analysis. Ratings were recoded as either: not self-referent ('not me'), or self-referent ('me'). Participants were subsequently asked to recall descriptors in a free recall task. A proportional score was calculated to account for the overall number of words recalled. Self-referent and non-self-referent conditions were calculated separately; positive scores reflect a positive bias whereas negative scores indicate a negative bias (Connolly, Abramson and Alloy, 2016).

Autobiographical Memory Test (AMT; Williams and Broadbent, 1986) 
Participants were presented visually and orally with five positive (relaxed, lucky, excited, relieved, loved) and five negative (hopeless, failure, sad, angry, lonely) cue words, in a randomised order, and asked to recall a specific memory. This was defined as "a memory of a particular event that occurred on a particular day which could be from a long time ago or very recently and could be something very important or something very ordinary". Participants were given 60 seconds to produce a memory, which they then verbally described under no time condition. This task was audio recorded and later coded for specificity. Participants were given two practice trials, where they were prompted to recall a specific event if their memory did not fit the criteria of a specific memory. Practice items were excluded from analysis. Responses to positive and negative cues were recorded as either specific (e.g. "My friend took me to the Manchester derby on my birthday.") or overgeneral (e.g. "During the summer holidays when there was no one around."). The total number of specific and overgeneral memories was calculated. This task has been extensively used within research and has previously demonstrated good psychometric properties (Griffith, Kleim, Sumner and Ehlers, 2012).

Ambiguous Scenarios Task for Depression in Adolescents: (AST; Orchard, Pass and Reynolds; 2016)

Participants were presented with 20 hypothetical ambiguous scenarios designed to assess interpretation bias in relation to depression, asked to consider each as happening to them and imagine the outcome. For example: "You see a man running down the street and think about why he is running". Participants were asked to write down their imagined outcome, and rate its pleasantness on a 9-point scale (1='extremely unpleasant', 9='extremely pleasant'). Orchard et al., (2016) 
demonstrated good psychometric properties of this task. Only written descriptions were included in the current analysis due to lower internal reliability of the rating scale in this sample $(\alpha=.68)$. Written descriptions were coded as positive, negative, neutral or mixed. Overall bias scores were calculated by subtracting number of negative responses from number of positive responses. Therefore, positive scores reflect a positive bias whereas negative scores indicate a negative bias.

\section{Facial Expression Recognition Task (Chan et al., personal communication)}

This computer task, based on a task employed by Chan et al., (2007) and adapted by Chan et al. (personal communication), presented five emotional expressions: anger, disgust, fear, happy and sad. Stimuli were morphed using Morpheus Photo Morpher v3.17 software, from $0-100 \%$ intensity at incremental increases of $10 \%$, with $0 \%$ reflecting a neutral expression and $100 \%$ reflecting full intensity of expression. In total 220 stimuli were presented in a random order across five blocks. Each face was presented for $500 \mathrm{~ms}$ against a black background preceded by a fixation cross of 100ms. Participants were asked to identify the emotion displayed by a key press. Prior to the experimental trials participants completed six practice trials, which were excluded from analyses. Mean accuracy of identification was computed across each emotion. An accuracy score was calculated for each emotion.

\section{Procedure}

This study was approved by University Research Ethics Committee and local educational authorities. Written consent from participants and parents for those under 16 was obtained. Participants completed measures of depression, anxiety, 
wellbeing, neuroticism, rumination, and dysfunctional attitudes online. Tasks assessing memory, interpretation and facial expression recognition tasks were conducted during a face-to-face interview with a trained psychologist either individually or in small groups. Self-report and cognitive tasks were conducted within the same week.

\section{Statistical Analyses}

Analyses were primarily conducted in IBM SPSS version 22 (SPSS Inc., USA). Three participants did not complete the facial recognition task. Due to a procedural error, item 13 from the BBC Well-being Scale was missing at random and therefore excluded and mean rather than total scores were employed for further analysis. The reliability of this scale remained high $(\alpha=.95)$.

Assumptions of normality were met. Despite variables being correlated collinearity and tolerance statistics were within accepted limits $(\mathrm{VIF}=1.50$ and tolerance $=0.61$; Field, 2009).

False discovery rate (FDR) correction for multiple comparisons was applied to control for familywise error rate (Benjamini and Hochberg, 1995).

Analysis comprised two stages. Initially, to identify salient predictors, backwardselimination regressions were employed based on a criterion of $F>=.100$. Subsequently, surviving variables were entered in a hierarchical regression to examine their contribution in relation to neuroticism. 
The impact of gender and age were assessed using t-test and Pearson's correlation, if tests demonstrated significant effects these variables were included in regression models.

Each regression model consisted of two steps to predict each dependent variable (depression, anxiety and wellbeing) in turn: 1. Neuroticism, 2. Cognitive variables surviving backwards removal regression. When age and gender were included within regression models, these did not impact any results and as such have been excluded as variables in order to preserve statistical power. Gender was included in models of anxiety as univariate tests indicated significant relationships between gender and anxiety.

\section{Results}

[insert table 1 here]

See Table 1 for demographic information and descriptive statistics.

\section{Age and Gender Differences}

Female participants reported higher levels of anxiety than male participants: $t(97)=2.83, p=.006$. No significant gender differences were demonstrated for depression $(t(97)=-1.22, p=.27)$ or wellbeing $(t(96)=1.85, p=.07)$. There were no 
significant correlation of age with depression $(r=.13, p=.20)$, anxiety $(r=.06, p=.55)$ or wellbeing $(r=-.13, p=.21)$.

\section{Correlations}

Pearson's correlation results, controlling for age and gender, are presented in Table 2 and Figure 1.

\section{[insert table 2 here]}

Pearson's correlations, controlling for age and gender, indicate a significant relationship between depression and anxiety and a negative relationship between depression and wellbeing. Neuroticism was positively correlated with depressive symptoms and anxiety, while negatively correlated with wellbeing. See Table 2 and Figure 1 for full results. Briefly, correlations demonstrated the expected pattern with higher depression and anxiety symptoms as well as lower wellbeing generally correlated with greater negative biases, reduced positive biases or more maladaptive processing. However, mean accuracy bias scores of facial emotion recognition and autobiographical memory bias scores were not significantly correlated with any other variables.

\section{[insert figure 1 here]}

\section{Backwards-Elimination Regression}


Variables surviving the regression model of depression were: rumination, dysfunctional attitudes and non-self-referential recall bias. Those surviving the regression model of anxiety were: ambiguous scenarios bias and rumination. The ambiguous scenarios task bias, self-referential recall bias, dysfunctional attitudes and accuracy of anger identification were included in the wellbeing regression model (Supplementary Material).

\section{Hierarchical Multiple Regression}

See Table 3.

\section{Depression}

In predicting depression, neuroticism was a significant predictor $\left(r^{2}=.40 p<0.001\right.$, $\beta=0.35)$. The addition of cognitive variables: rumination $(\beta=0.26)$, dysfunctional attitudes $(\beta=-0.19)$ and self-referential 'not me' recall scores $(\beta=0.17)$, resulted in an increase of explained variance $\left(\Delta r^{2}=.12, p<0.005 ; r^{2}=.52, F(4,94)=25.49, p<0.001\right)$. All included variables demonstrated significant beta values.

\section{Anxiety}

In the first block gender alone significantly predicted anxiety $\left(r^{2}=.08, p<0.05\right)$, as did the addition of neuroticism $\left(r^{2}=.54, p<0.001, \beta=0.55\right)$. Inclusion of cognitive variables (ambiguous scenarios task bias $(\beta=-\mathbf{0 . 1 3})$ and rumination $\beta=0.21)$ demonstrated a small but significant increase of explained variance $\left(\Delta r^{2}=.05\right.$ and $r^{2}=.59$; $F(3,95)=44.46, p<.001$,$) . Only neuroticism and rumination demonstrated significant$ beta values.

\section{Wellbeing}


In the first block of the model of wellbeing, neuroticism significantly predicted wellbeing ( $\left.\mathrm{r}^{2}=.36, \beta=-0.32, \mathrm{p}<0.001\right)$. Inclusion of cognitive variables: ambiguous scenarios task bias $(\beta=0.19)$, self-referential recall 'me' bias $(\beta=0.18)$, self-referential recall 'not-me' bias $(\beta=-\mathbf{0 . 1 6})$, dysfunctional attitudes $(\beta=0.17)$, and recognition of angry facial expressions $(\beta=-\mathbf{0 . 1 4})$, demonstrated a significant $r^{2}$ change $\left(\Delta r^{2}=.19\right.$, $\left.\mathrm{p}<0.001 ; \mathrm{r}^{2}=.55, \mathrm{~F}(6,89)=17.74, \mathrm{p}<0.001\right)$. Only neuroticism, ambiguous scenarios task bias and self-referential recall 'me' bias demonstrated significant beta values.

\section{[insert table 3]}

\section{Discussion}

Rumination, dysfunctional attitudes, and negative biases in ambiguous scenarios interpretation and self-referential memory, significantly predicted depression in adolescents above and beyond neuroticism. Neuroticism predicted around $40 \%$ of the variance of depression symptoms, $54 \%$ of anxiety symptoms and $35 \%$ of wellbeing, in line with previous research (e.g. Kotov et al., 2010 and Bartels et al., 2013). As hypothesised, adding cognitive variables significantly increased the explained variance of depression by $12 \%$, anxiety by $5 \%$ and wellbeing by $19 \%$. This supports previous findings that have associated negative cognitive biases with psychological outcomes. Orchard (2016) demonstrated that biased interpretation was most negative for adolescents with a depression diagnosis and most positive for non-depressed controls. The current study highlights the salience of interpretation bias across psychological distress and wellbeing. 
In an undergraduate student sample, Rude et al., (2002) demonstrated that including a measure of interpretation under a cognitive load condition increased predictions of depression scores by $11 \%$ for male participants and $0 \%$ for female participants. This increase is in line with the current findings; however, we did not identify such gender disparity. Rude et al. (2002) suggest that such divergence may be due to gender bias in self-report or that despite decreased negative biases in women, their subsequent behaviour or processing (such as rumination) increases their vulnerability to experience depression.

This study demonstrates, similar to findings of adult populations, neuroticism to be a key predictor of mental health outcomes (Lahey, 2009). Neuroticism was the strongest predictor of each mood variable; each standard deviation increase of MFQ was associated with a 0.55 increase of neuroticism. This magnitude is similar to findings of a meta-analysis of 33 population based samples (Malouff, Thorsteinsson and Schutte, 2005). The predictive power of neuroticism cannot be overlooked as it indicates underlying biopsychological components of depressive disorders worthy of further neurobiological study to assess its expression and mechanism of action.

Like Zupan et al., (1987), this study indicates that greater depressive symptomology and anxiety was strongly correlated with a bias towards recalling negative selfreferenced words. However, for non-self-referential words, higher levels of depression and anxiety were related to recalling more positive adjectives. This suggests that symptoms of depression and anxiety are not associated with global 
negative biases, but a specific negative bias in relation to the self. This is consistent with findings of more negative self-perceptions in young people at risk for depression (Chan et al., 2007). Previous research has identified a self-positivity bias within healthy individuals; whereby, individuals are more likely to overestimate their own success compared to the success of others. However, depressed individuals have been shown to lack such positivity bias (Alloy and Ahrens, 1987). Self-concept and self-referent bias may be of particular significance within adolescent populations. Adolescence is a sensitive period were self-concept is developing (Marcia, 1980), biases towards negative self-descriptors at this age group may impair the development of adaptive self-concepts.

Regression models predicting each outcome variable included different predictors, signaling salience of distinct cognitive bias in depression, anxiety and wellbeing. Specifically, dysfunctional attitudes emerged as a significant predictor of depression and wellbeing. Robinson and Alloy (2003) found that dysfunctional attitudes and components of rumination interacted to prospectively predict onset, number and duration of depressive episodes, in an undergraduate sample. In the present study, dysfunctional attitudes were found to be more strongly related to depression and wellbeing than anxiety. This is expected as the dysfunctional attitudes scale was developed to capture thinking styles associated with depression (Power et al., 1994). Rumination is a significant research focus and has been frequently related to emotional disorders (Young and Dietrich, 2015). Consistent with this, our findings demonstrate that rumination predicted depressive and anxiety symptoms but was less strongly associated with wellbeing. 
No significant relationships between autobiographical memory recall and depression, anxiety or wellbeing were demonstrated. Previous findings have been inconsistent. Chan et al., (2007) demonstrated no significant differences in this task between individuals at high vs. low risk for depression by virtue of neuroticism. Further, Swales et al. (2010) found group differences in specificity of autobiographical memory when comparing clinical groups to controls, but that this was due to individuals within clinical groups recalling the same suicide related memories in response to multiple cue words. However, autobiographical memory impairment in depressed groups is a robust finding with large effect sizes within adult samples (Williams et al., 1997). The above mixed findings may indicate that differences in autobiographical memory are related to symptom severity; such bias may be a scar effect rather than an antecedent risk; or that, contrary to adult research, overgeneral memory is not a reliable cognitive marker of adolescent depression.

This study demonstrated that accuracy in identification of angry faces predicted wellbeing. This indicates that individuals with lower wellbeing were better able to recognise anger, suggesting higher sensitivity towards negative facial expressions. This is consistent with previous research which found that recognition accuracy of negative emotions to be associated with depressive relapse (Bouhuys et al., 1999). This finding supports the protective value of wellbeing in that, the association with a positivity bias in facial emotion identification may foster positive social interactions. However, the effect size of our finding was small and no other significant relationships between psychological outcomes and facial expressions were demonstrated. Previous research demonstrated inconsistencies which may indicate that facial emotion recognition bias is not a robust marker of mood disorders. Smith et al., 
Alternatively, there may be a task failure to detect subtle differences in interpretation. Picci and Scherf (2016) showed that adolescents were significantly better at identifying faces of their own age and hypothesised a 'dip' in facial recognition whereby there is a recalibration of the face-processing system away from caregivers towards peers. It is possible that the use of adult faces has interfered with an effect of emotion. Review of adult studies of facial emotion interpretation indicates evidence supporting an increased tendency to interpret facial expressions more negatively (Bourke, Douglas and Porter, 2010). However, like within adolescent samples, there is discrepancy with some evidence indicating a global deficit of facial emotional processing, while some studies support a mood-congruent bias towards negative emotions in depressed groups.

A number (18\%) of participants reached scores associated with clinical depression on the MFQ (Daviss et al., 2006), consistent with prevalence estimates within this age group (Avenevoli et al., 2015). This indicates the representative nature of the sample. A key strength of this study has been the assessment of wellbeing. The mental health spectrum ranges from highly disabling disorder to positive states of wellbeing. Components of wellbeing are recognised protective factors against disorders (NHS Scotland, 2016). Our results highlight the importance of cognitive biases for subjective wellbeing. Aiming to enhance positivity bias in order to boost wellbeing, potentially protecting against depression or anxiety in preventative or early intervention strategies, may be an avenue of future research. Future research may investigate the potential to develop interventions that address specific biases, relevant to individuals' experiences and symptoms, particularly in light of the importance of cognitive contributions (rumination, dysfunctional attitudes, self- 
referent processing and interpretation biases). Similarly, development of interventions addressing cognitive bias in order to enhance wellbeing, which is of importance for quality of life, has been associated with favourable life outcomes, including longevity (Sadler et al., 2011).

There are some limitations of this study; primarily, predictions are based on regression analyses of cross-sectional data, limiting the ability to conclude causality. Similarly, age has been demonstrated to exert non-linear development which has not been accounted for within this study. Self-report questionnaires have been employed rather than clinical interviews. The latter was deemed less feasible due to issues of anonymity, confidentiality and the non-clinical nature of the sample. Further, although the impact of neuroticism has been analysed as distinct to cognitive factors, it is possible that neuroticism itself is influenced by mood state and biases, which may impact interpretations of the current results. Within this study, including neuroticism in initial blocks has allowed for the explanation of unique variance by cognitive biases. In future work a larger sample may be employed to allow for full mediation analysis to examine such effects.

To examine a wide range of mood states, participants were recruited from community settings, therefore results are less generalizable to clinical groups but are representative of typical adolescents. Recruiting adolescents is notoriously difficult. While the hierarchical regression models were sufficiently powered, initial identification of salient variables was underpowered and as such, variables with smaller effect sizes may not have been identified. Finally, our regression models 
explained approximately $50 \%$ of the variance of depression, anxiety and wellbeing, indicating that there are important factors that have not been accounted for here. For example, stressful life events and general cognitive performance would be valuable factors to include in future studies.

\section{Conclusion}

This study assessed a relatively wide range of cognitive biases within a single sample using validated and standardized measures in combination with experimental paradigms to demonstrate that cognitive biases accounted for variability in depression, anxiety and wellbeing over and above that of neuroticism. Results stress the importance of cognitive factors in symptoms of depression, anxiety and wellbeing. Contributions of cognitive mechanisms, identified here, are a feasible target for behavioural and cognitive modification and improvement of interventions, potentially targeting specific biases and to enhance wellbeing as a protective factor are worthy of further study. 


\section{References}

Alloy, L.B., and Ahrens, A.H., (1987) Depression and pessimism for the future: biased use of statistically relevant information in predictions for self versus others, Journal of Personality and Social Psychology, 52(2):366-378

Angold, A., Costello, E.J., Messer, S.C., Pickles, A., Winder, F., and Silver, D., (1995) Development of a short questionnaire for use in epidemiological studies of depression in children and adolescents. International Journal of Methods in Psychiatric Research, 5:237-249

Avenevoli, S., Swendsen, J., He, J.P., Burstein, M., and Merikangas, K.R., (2015), Major Depression in the national Comorbidity Survey- Adolescent Supplement: Prevalence, Correlates, and Treatment. Journal of the American Academy of Child and Adolescent Psychiatry, 54(1):37-44.e2

Bartels, M., Cacioppo, J.T., van Bejisterveldt, T.C., Boomsma, D.I., (2013), Exploring the association between well-being and psychopathology in adolescents, Behavior Genetics, 43(3):177-190

Benjamini, Y., and Hochberg, Y., (1995), Controlling the false discovery rate: a practical and powerful approach to multiple testing, Journal of the Royal Statistical Society. Series B(Methodological), 57(1):289-300

Bouhuys, A.L., Geers, E., Gordijn, M.C.M., (1999) Depressed patients' perceptions of facial emotions in depressed and remitted states are associated with relapse: a longitudinal study, Journal of Nervous and Mental Disease, 187:595-602

Bourke, C., Douglas, K., Porter, R., (2010) Processing of facial emotion expression in major depression: a review, Australian and New Zealand Journal of Psychiatry, 44(8):681-696 
Chan, S., Goodwin, G., and Harmer, C., (2007) Highly neurotic never-depressed students have negative biases in information processing, Psychological Medicine, $37(9): 1281-1292$

Connolly, S.L., Abramson, L.Y., and Alloy, L.B., (2016) Information processing biases concurrently and prospectively predict depressive symptoms in adolescents: Evidence from a self-referent encoding task, Cognition and Emotion, 30(3):550-60

Daviss, W.B., Birmaher, B., Melhem, N.A., Axelson, D.A., Michaels, S.M., and Brent, D.A., (2006) Criterion validity of the Mood and Feelings Questionnaire for depressive episodes in clinic and non-clinic subjects. Journal of Child Psychology and Psychiatry, 47(9):927-934

Dunn, V., and Goodyer, I.M., (2006) Longitudinal investigation into child-and adolescenceonset depression: psychiatric outcome in early adulthood, The British Journal of Psychiatry, 188(3):216-222

Eysenck, S.B.G., Eysenck, H.J., and Barrett, P., (1985) A revised version of the psychoticism scale, Personality and Individual Differences, 6:21-29

Everaert, J., Duyck, W., and Koster, E.H.W., (2015), Emotionally Biased Cognitive Processes: The weakest link predicts prospective changes in depressive symptom severity, PLoS ONE 10(5):e0124457.

Field, A., (2009), Discovering Statistics Using SPSS, $3^{\text {rd }}$ Edition, Sage Publications Ltd, London

Goodyer, I.M., Reynolds. S., Barrett, B., Byford, S., Dubicka, B., Hill, J., ..Fonagy, P., (2017) Cognitive behavioural therapy and short term psychoanalytical intervention in adolescents with unipolar major depressive disorder (IMPACT): a multicentre, 
pragmatic, observer-blind randomised controlled superiority trial, The Lancet Psychiatry, 4(2):109-119

Griffith, J.W., Kleim, B., Sumner, J.A., and Ehlers, A., (2012) The factor structure of the autobiographical memory test in recent trauma survivors, Psychological Assessment, 24(3):640-646.

Hettema, J.M., Neale, M.C., Myers, J.M., Prescott, C.A., and Kendler, K.S., (2006) A population-based twin study of the relationship between neuroticism and internalizing disorders, American Journal of Psychiatry, 163(5):857-864

Joormann, J., and Vanderlind, M.W., (2014) Emotional Regulation in Depression. The role of biased cognition and reduced cognitive control, Clinical Psychological Science 2(4):402-421

Kelvin, R.G., Goodyer, I.M., Teasdale, J.D., and Brechin, D., (1999) Latent negative selfschema and high emotionality in well adolescents at risk for psychopathology, Journal of Child Psychology and Psychiatry, 40(6):959-968

Kinderman, P., Schwannauer, M., Pontin, E., and Tai, S., (2011) The development and validation of a general measure of well-being: the BBC well-being scale, Quality of Life Research, 20(7):1035-1042

Klein, D.N., Kotov, R., and Bufferd, S.J., (2011) Personality and depression: Explanatory models and review of the evidence, Annual Review of Clinical Psychology, 7:269295

Kotov, R., Gamez, W., Schmidt, F., and Watson, D., (2010), Linking "big" personality traits to anxiety, depressive, and substance use disorders: A meta-analysis, Psychological Bulletin, 136(5):768-821 
Lahey, B.B., (2009) Public Health Significance of Neuroticism, The American Psychologist, $64(4): 241-256$

Lee, A., and Hankin, B.L., (2009) Insecure attachment, dysfunctional attitudes, and low self-esteem predicting prospective symptoms of depression and anxiety during adolescence, Journal of Clinical Child and Adolescent Psychology, 28(2):219-231

Lucinao, M., Hagenaars, S.P., Davies, G., Hill, W.D., Clarke, T-K., Shirali, M., ... and Deary, I.J., (2018), Association analysis in over 329,000 individuals identifies 116 independent variants influencing neuroticism, Nature Genetics, 50:6-11

Malouff, J.M., Thorsteinsson, E.B., and Schutte, N.S., (2005) The relationship between the five-factor model of personality and symptoms of clinical disorders: a metaanalysis, Journal of Psychopathology and Behavioral Assessment, 27(2):101-114

Marco, E.M., Marci, S., and Laviola, G., (2011) Critical Age Windows for Neurodevelopmental Psychiatric Disorders: Evidence from Animal Models, Neurotoxicity Research, 19(2):286-307

Marcia, J.E., (1980) Identity in adolescence in Adelson, J., (Ed.) Handbook of Adolescent Psychology, 159-187

McLaughlin, K.A., and Nole-Hoeksema, S., (2011) Rumination as a transdiagnostic factor in depression and anxiety, Behavioural Research Therapy, 49(3):186:93

Merikangas, K.R., He J.P., Burstein, M., Swanson, S.A., Avenevoli, S., Cui, L., ... and Swendsen, J., (2010) Lifetime Prevalence of Mental Disorders in U.S. Adolescents: Results from the National Comorbidity Survey Replication, Journal of the American Academy of Child and Adolescent Psychiatry, 49(10):980-989 
Micco, J.A., Henin, A., Hirshfeld-Becker, D.R., (2014) Efficacy of interpretation bias modification in depressed adolescents and young adults, Cognitive Therapy Research, 38(2):89-102

Mor, N., and Winquist, J., (2002) Self-focused attention and negative affect: A metaanalysis, Psychological Bulletin, 128(4):638-62

MQ (2016), Young People's Mental Health.

https://www.mamentalhealth.org/mental-health/children-and-young-people, on $09 / 10 / 17$

Navrady, L.B., Ritchie, S.J., Chan, S.W.Y., Kerr, D.M., Adams, M.J., Hawkins, E.H., ... and Mclntosh, A.M., (2017) Intelligence and neuroticism in relation to depression and psychological distress: Evidence from two large population cohorts, European Psychiatry, 43:58-65

NHS Health Scotland (2016), Good Mental Health For All, ISBN: 978-1-84485-592-6, http://www.healthscotland.scot/media/1091/good-mental-health-for-all-feb-2016.pdf, on $06 / 10 / 17$

National Institute for health and Clinical Excellence, (2005), Depression in children and young people: identification and management in primary, community and secondary care, London, England

Nolen-Hoeksema, S., (2000). The role of rumination in depressive disorders and mixed anxiety/depressive symptoms. Journal of Abnormal Psychology, 109(3):504-511. 
Orchard, F., Pass, L., and Reynolds, S., (2016) 'It was all my fault'; Negative interpretation bias in depressed adolescents, Journal of Abnormal Child Psychology, $44(5): 991-8$

Picci,G., and Scherf, K.S., (2016) From Caregivers to Peers: Puberty shapes human face perception, Psychological Science 27(11):1461-1473

Platt, B., Waters, A.M., Schulte-Koerne, G., Engelmann, L., and Salemink, E., (2017) A Review of Cognitive biases in youth depression: attention, interpretation and memory, Cognition and Emotion, 31(3):462-83

Power, M.J., Katz., R., McGuffin, P., Duggan, C.F., Lam, D., and Beck, A.T., (1994) The Dysfunctional Attitude Scale (DAS): A comparison of forms A and B and proposals for a new subscaled version, Journal of Research in Personality, 28(3):263-276

Robinson, M.S., and Alloy, L.B., (2003) Negative cognitive styles and stress-reactive rumination interact to predict depression: A prospective study, Cognitive Therapy and Research, 27(3):275-291

Rude, S.S., Wenzlaff, R.M., Gibbs, B., Vane, J., and Whitney, T., (2002) Negative processing biases predict subsequent depressive symptoms, Cognition and Emotion, $16(3): 423-440$

Sadler, M.E., Miller, C.J., Christensen, K., and McGue, M., (2011) Subjective well-being and longevity: A cotwin control study, Twin Research and Human Genetic: The Official Journal of the International Society for Twins, 14(3):249-256 
Schepman, K., Taylor, E., Collishaw, S., and Fombonne, E., (2012) Face emotion processing in depressed children and adolescents with and without comorbid conduct disorder, Journal of Abnormal Child Psychology, 40(4):583-93

Spence, S., (1998), A measure of anxiety symptoms among children, Behaviour Research and Therapy, 3:545-566

Sumner, J. A., Griffith, J. W., Mineka, S., Rekart, K. N., Zinbarg, R. E., and Craske, M. G. (2011), Overgeneral autobiographical memory and chronic interpersonal stress as predictors of the course of depression in adolescents, Cognition and Emotion, 25:183-192

Swales, M.A., Williams, J.M.G., Wood, P., (2010), Specificity of autobiographical memory and mood disorder in adolescents, Cognition and Emotion, 15(3):321-331

Tang, T.Z., DeRubeis, R.J., Hollon, S.D., Amsterdam, J., Shelton, R., and Schalet, B., (2009) A placebo-controlled test of the effects of paroxetine and cognitive therapy on personality risk factors in depression, Archives of General Psychiatry, 66(12):13221330

Timbremont, B., Braet, C., Bosmans, G., and Van Vlierberghe, L., (2008) Cognitive biases in depressed and non-depressed referred youth, Clinical Psychology and Psychotherapy, 15(5):329-339

Treynor, W., Gonzalez, R., and Nolen-Hoeskema, S., (2003), Rumination Reconsidered: A psychometric analysis, Cognitive Therapy and Research, 27(3):247-259

Wang, P.S., Berglund, P., Olfson, M., Pincus, H.A., Wells, K.B., and Kessler, R.C., (2005) Failure and delay in initial treatment contact after first onset of mental 
disorders in the new comorbidity survey replication, Archives of General Psychiatry, 62(2):603-613

Williams, J.M.G., and Broadbent, K., (1986) Autobiographical memory in suicide attempters, Journal of Abnormal Psychology, 95:144-149

Williams, J.M.G., Watts, F.N., MacLeod, C., and Matthews, A., (1997) Cognitive Psychology and Emotional Disorders (second edition), Wiley and Sons, England

World Health Organisation, (2013), Mental Health Action Plan: 2013-2020, ISBN: 97892 4150602

World Health Organisation, (2017) Depression Fact Sheet, http://www.who.int/mediacentre/factsheets/fs369/en/, Accessed on: 06/10/2017

Wray, N.R., Birley, A.J., Sullivan, P.F., Visscher, P.M., and Martin (2007) Genetic and phenotypic stability of measures of neuroticism over 22 years, Twin Research and Human Genetics, 10(5):695-702

Young, C.C., and Dietrich, M.S., (2015) Stressful life events, worry and rumination predict depressive and anxiety symptoms in young adolescents, Journal of Child and Adolescent Psychiatric Nursing, 28(1):35-42

Zupan, B.A., Hammen, C., and Jaenicke, C., (1987) The effects of current mood and prior depressive history on self-schematic processing in children, Journal of Experimental Child Psychology, 43(1):149-58 
Table 1: Descriptive Statistics

\begin{tabular}{lrrrr}
\hline & Mean & Std. Deviation & Minimum & Maximum \\
\hline Age & 14.94 & 1.52 & 13 & 18 \\
MFQ (Depression) & 15.26 & 12.88 & 0 & 57 \\
SCAS (Anxiety) & 27.59 & 17.09 & 2 & 95 \\
BBC (Wellbeing) & 68.56 & 12.87 & 35 & 92 \\
Neuroticism & 6.24 & 3.41 & 0 & 12 \\
RRS (Rumination) & 21.11 & 6.41 & 10 & 40 \\
DAS (Dysfunctional Attitudes Scale) & 91.55 & 17.45 & 51 & 168 \\
AMT: Autobiographical Memory Task (Specific) & 7.36 & 1.95 & 2 & 10 \\
AMT: Autobiographical Memory Task (Overgeneral) & 2.04 & 1.66 & 0 & 7 \\
AST: Ambiguous Scenarios Task Bias & 1.33 & 5.76 & -15 & 15 \\
SRR: Self-Referential Recall 'Me' & 0.33 & 0.29 & -0.50 & 1 \\
SRR: Self-Referential Recall 'Not Me' & -0.23 & 0.19 & -0.56 & 0.50 \\
Facial Expression Recognition: Anger & 0.57 & 0.15 & 0.23 & 0.88 \\
Facial Expression Recognition: Disgust & 0.43 & 0.15 & 0.05 & 0.73 \\
Facial Expression Recognition: Fear & 0.60 & 0.12 & 0.25 & 0.80 \\
Facial Expression Recognition: Happy & 0.76 & 0.08 & 0.48 & 0.93 \\
Facial Expression Recognition: Sad & 0.67 & 0.20 & 0.15 & 0.95 \\
\hline
\end{tabular}

Smith et al., 
Table 2: Pearson's Correlation Coefficient (r) between outcome variables and cognitive variables, controlling for age and gender

\begin{tabular}{|c|c|c|c|c|c|c|c|c|c|c|c|c|c|}
\hline & 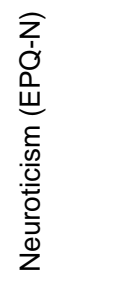 & 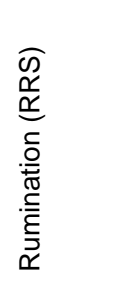 & 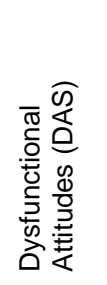 & 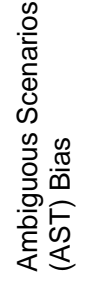 & 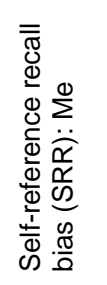 & 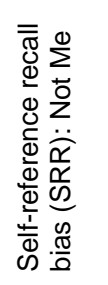 & 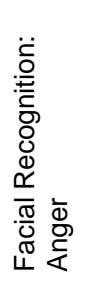 & 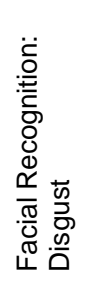 & 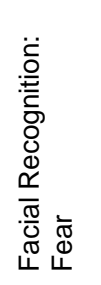 & 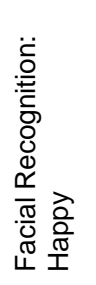 & 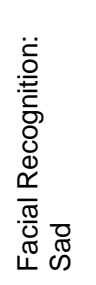 & 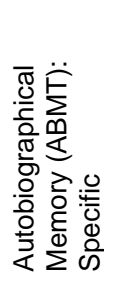 & 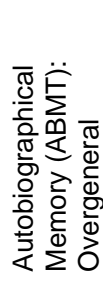 \\
\hline MFQ & $0.63^{* *}$ & $0.58^{* *}$ & $-0.51^{* \star}$ & $-0.41^{\star *}$ & $-0.35^{\star *}$ & $0.42^{* \star}$ & 0.21 & 0.01 & 0.14 & -0.10 & -0.07 & -0.06 & 0.01 \\
\hline SCAS & $0.71^{\star *}$ & $0.57^{\star *}$ & $-0.48^{* *}$ & $-0.41^{\star *}$ & $-0.32^{*}$ & $0.34^{* *}$ & 0.17 & -0.08 & 0.08 & -0.15 & -0.15 & 0.00 & -0.07 \\
\hline BBC & $-0.57^{\star *}$ & $-0.48^{\star *}$ & $0.51^{\star *}$ & $0.48^{\star *}$ & $0.45^{\star *}$ & $-0.48^{\star *}$ & -0.27 & 0.07 & -0.02 & 0.16 & 0.03 & 0.04 & -0.04 \\
\hline
\end{tabular}

Note: ${ }^{*}$ indicates significance at $\mathrm{p}<0.001 ;{ }^{*}$ indicates significance at $\mathrm{p}>0.05$. MFQ refers to the Mood and Feelings Questionnaire, SCAS refers to Spence Child Anxiety Scale, BBC refers to the BBC Well-being Scale, 
Table 3: Hierarchical Regression Models of Mood Variables

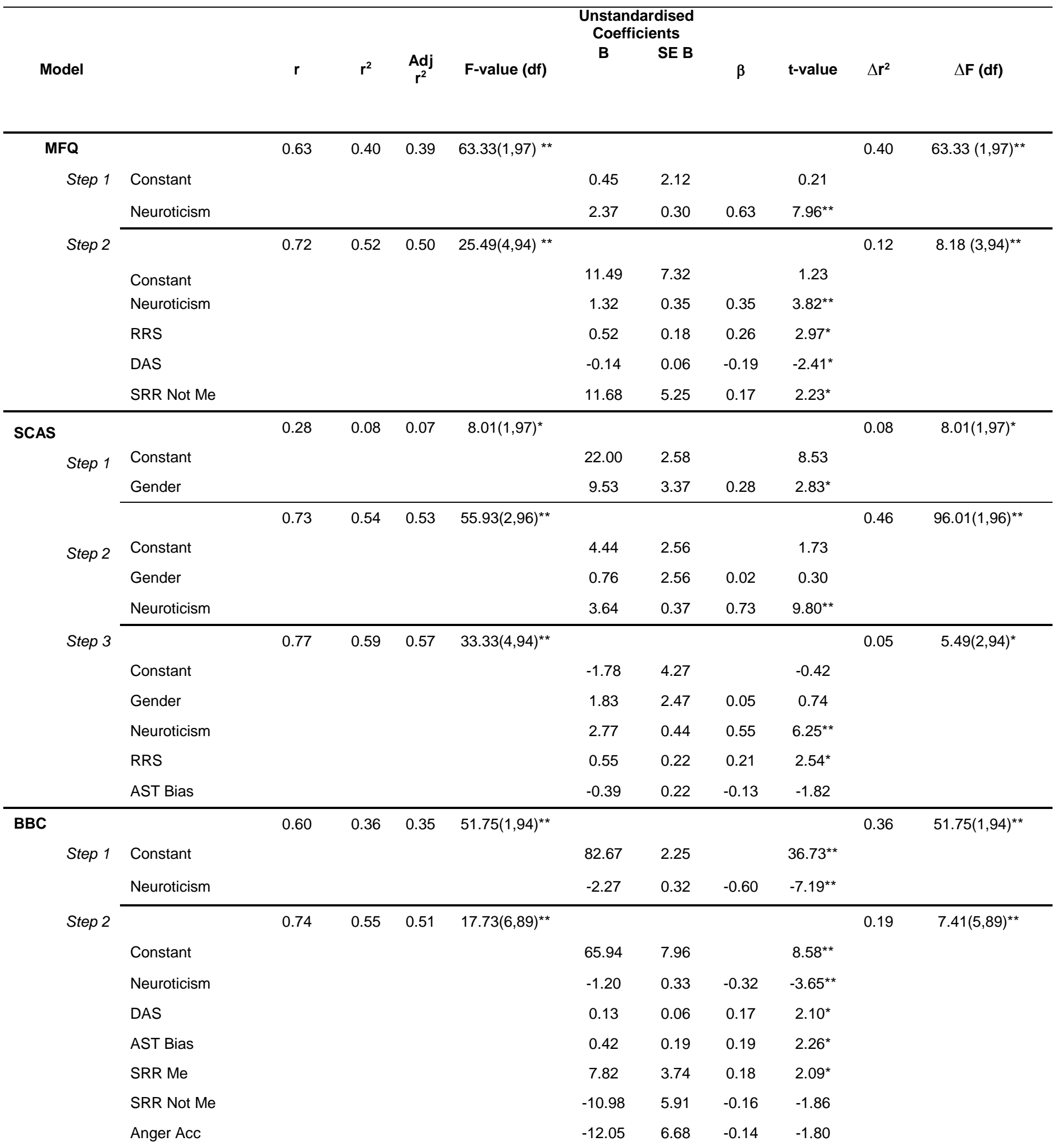

Note: **indicates significance at $p<0.001$; *indicates significance at $p>0.05$. MFQ refers to the Mood and Feelings

Questionnaire, SCAS refers to Spence Child Anxiety Scale, BBC refers to the BBC Well-being Scale, DAS refers to the Dysfunctional Attitudes Scale, RRS refers to the Ruminative Response Scale, AST refers to the Ambiguous Scenarios Scale, SRR refers to the Self-Referential Recall task. Anger Accuracy refers to the proportional accuracy of the facial emotional expression questionnaire. 


\section{Correlation Map: Strength of Association}

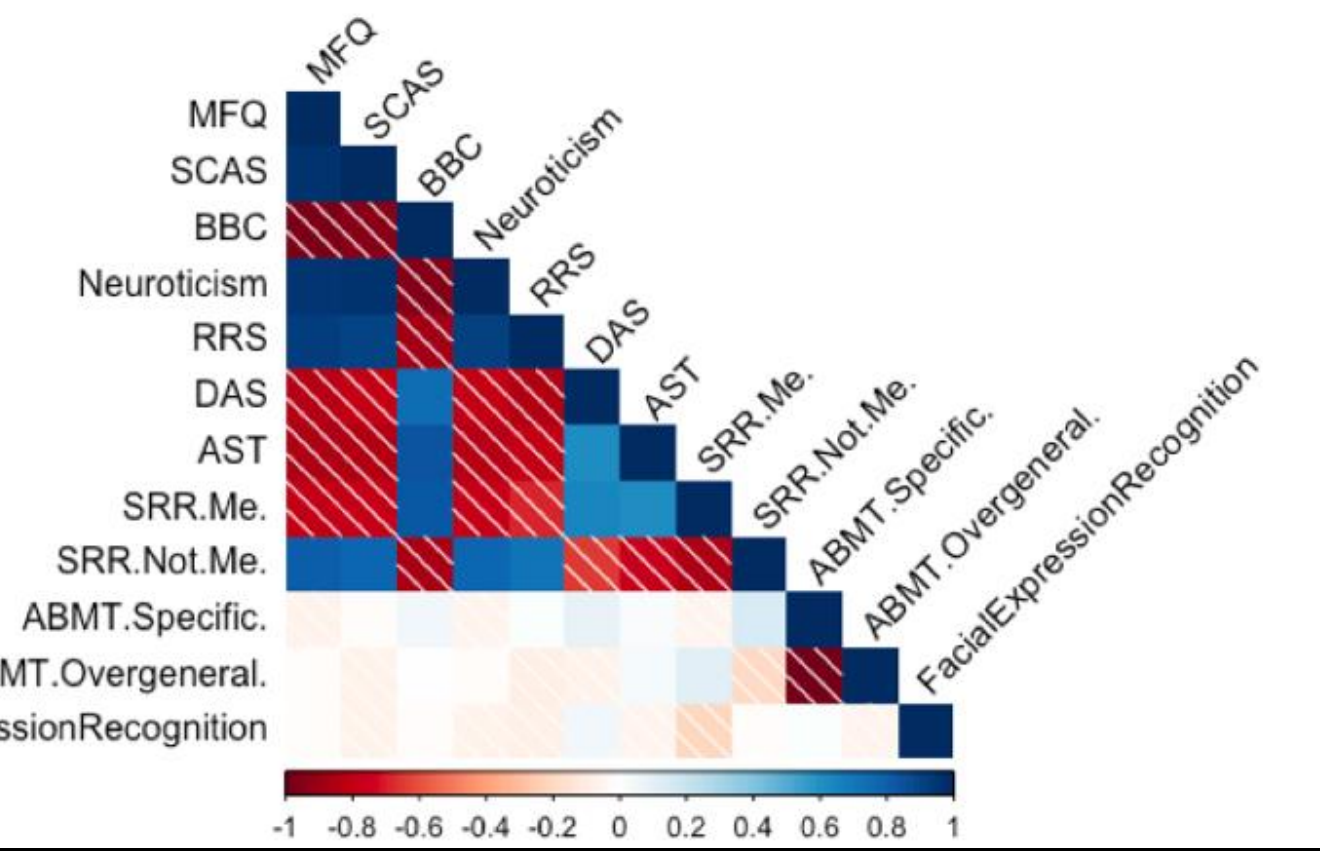

Figure 1: Strength of Association Heat Map

Note: Figure depicts Pearson correlation between variables. For simplicity, Facial Emotion Recognition Bias is represented by a mean across emotions. Diagonal stripes indicate negative correlation. MFQ refers to the Mood and Feelings Questionnaire, SCAS refers to Spence Child Anxiety Scale, BBC refers to the BBC Well-being Scale, DAS refers to the Dysfunctional Attitudes Scale, RRS refers to the Ruminative Response Scale, AST refers to the Ambiguous Scenarios Scale, SRR refers to the Self-Referential Recall task. ABMT refers to the Autobiographical Memory Test 